\title{
De la ficción a la realidad: órganos-en-chips al Servicio de la Ciencia y la Medicina
}

\author{
Voyage from fiction to reality: organs-on-chips \\ in the Service of Science and Medicine
}

\author{
Marina Macías Silva*
}

Los órganos-en-chips (organs-on-chips) son dispositivos fabricados a escalas micrométricas, se han descrito como sistemas biomiméticos que tienen como función principal lograr el mantenimiento de la unidad funcional de un órgano vivo en una estructura tridimensional (3D). Así, la tecnología inspirada en la biología, ha llevado a la fabricación de estos dispositivos, los cuales consisten en diminutos canales 3D microfluídicos, fabricados con un material polimérico transparente revestido con células, con el fin de replicar aspectos importantes de un órgano, como la micro-arquitectura 3D que se da por la distribución en el espacio de diferentes tejidos, las interfaces funcionales entre tejido-tejido y los complejos microambientes bioquímicos y mecánicos de los órganos. Así, estos dispositivos conocidos como órganos-en-chips, recapitulan la complejidad de los órganos vivos, en cuanto a su arquitectura, microambiente y fisiología.

Los procesos celulares que han podido ser estudiados en este tipo de dispositivos son muy diversos, entre ellos están el crecimiento y la muerte, la morfología de células y tejidos, la diferenciación, la motilidad y contractilidad de las células, la función de las barreras tisulares, el metabolismo, la secreción, el estrés oxidativo, entre otros. Además, se han realizado investigaciones sobre algunos procesos biológicos normales o patológicos, como la transición epitelio-mesénquima, la contracción cardiaca, el metabolismo de fármacos, la vasculogénesis, la angiogénesis tumoral, la invasión y metástasis de células cancerosas, la eficacia y los efectos colaterales de algunos fármacos, la mecanobiología, entre otros.

La mecanobiología es un proceso por el cual las células convierten señales mecánicas en respuestas biológicas. Este proceso es iniciado por diversos estímulos mecánicos como el estrés laminar, el flujo intersticial, o la tensión ejercida por la matriz extracelular. Dichos estímulos provocan diferentes respuestas biológicas en las células o tejidos, como por ejemplo: una expresión diferencial de genes, la secreción de proteínas o la migración celular. Es común que estas respuestas biológicas involucren cambios en las propiedades mecánicas intrínsecas de las células, como por ejemplo la generación de cambios en la rigidez del citoesqueleto. Además, en ocasiones, la mecanotransducción involucra procesos de retroalimentación, es decir, que los cambios intracelulares pueden modificar las señales mecánicas externas; un caso de retroalimentación se presenta, por ejemplo, cuando un estrés por flujo laminar (señal mecánica externa) causa que las células se polaricen o se alarguen (respuesta biológica), lo cual lleva a una alteración en el flujo del fluido y al mismo tiempo a una alteración en el estrés laminar que es impartido sobre la monocapa epitelial. Para investigar procesos como la mecanobiología, se desarrollan cultivos celulares que utilizan los avances en la tecnología de la microfabricación y la microfluídica para controlar el microambiente celular y mimetizar el contexto fisiológico. A la vanguardia en este tipo de desarrollo tecnológico se encuentran los dispositivos tipo órganoen-un-chip, en éstos se intenta crear el modelo más sencillo que sea posible para recapitular las respuestas fisiológicas bajo estudio. Es importante mencionar que el desarrollo de estas tecnologías requiere de alianzas entre los ingenieros, los físicos, los biólogos, los biólogos computacionales y los clínicos para implementar las metodologías que permitan capturar los componentes esenciales de la fisiología de los órganos de interés.

Otro de los aspectos que se debe destacar es la ventaja que representan los modelos de cultivo 3D

\footnotetext{
* Instituto de Fisiología Celular de la Universidad Nacional Autónoma de México.
}

Este artículo puede ser consultado en versión completa en http://www.medigraphic.com/facultadodontologiaunam 
que, a diferencia de los cultivos 2D, permiten recapitular in vivo las complejas interacciones entre diferentes tipos celulares que son reguladas por varios factores, como citocinas, nutrientes, factores de crecimiento, hormonas, la matriz extracelular y las uniones intercelulares. En los órganos-en-chips es posible controlar desde el tipo de sustrato para cultivar a las células hasta el movimiento de los fluidos en el cultivo, para así reproducir las señales mecánicas inducidas por los flujos fisiológicos (por ejemplo, el flujo sanguíneo y el flujo intersticial), así como la deformación tisular (por ejemplo, la respiración, la peristalsis y el latido cardiaco), incluyendo además el estrés por flujo laminar, la tensión, la compresión y la torsión. Así, en años recientes, estos sistemas biomiméticos han sido utilizados para establecer modelos que recapitulen la complejidad funcional y estructural de algunos órganos humanos, como el hígado, corazón, intestino, pulmón, riñón, cerebro y huesos. Actualmente, los científicos también han empezado a desarrollar modelos multi-órganos, capaces de duplicar procesos secuenciales que, por ejemplo, podrían influir en la actividad de los fármacos en el cuerpo; así, estos nuevos sistemas llamados cuerpo-en-unchip (body-on-a-chip) están diseñados para mimetizar mejor la complejidad a nivel de un órgano y de las interacciones órgano-órgano.

En el desarrollo tecnológico de los órganos-enchips, uno de los retos técnicos que se ha presentado está relacionado con la elección de los tipos de materiales usados en su fabricación, entre los cuales se encuentran el policarbonato, el poliestireno, y un tipo de silicona llamado PDMS (polimetilsiloxano). Estos materiales son usados como sustratos de cultivo; sin embargo, sus propiedades fisicoquímicas desafortunadamente no mimetizan a las matrices extracelulares in vivo. EI PDMS es uno de los materiales más usados en la fabricación de estos dispositivos, y a pesar de las ventajas que representa su uso, se ha visto que tiene algunas deficiencias, como por ejemplo la capacidad de absorber pequeñas moléculas, lo que podría disminuir las concentraciones efectivas de los fármacos y disminuir su actividad en los cultivos realizados en los microdispositivos. Algunos estudios han mostrado que cuando se comparan los cultivos celulares realizados con métodos tradicionales con los cultivos en los dispositivos microfluídicos, se pueden observar diferencias significativas en cuanto a la proliferación celular y a la actividad metabólica de las células (consumo de oxígeno, síntesis de proteínas, etc.). Por lo tanto, actualmente se están implementando técnicas para modificar químicamente las superficies del PDMS, y al mismo tiempo se está explorando el uso de materiales alternativos, para así poder establecer las mejores condiciones de cultivo en este tipo de microdispositivos y evitar cualquier cambio en el fenotipo o en el contexto celular.

Por otro lado, también se está trabajando en la integración de los órganos-en-chips a plataformas bio-analíticas que incluyan sensores y óptica miniaturizada, para poder realizar análisis bioquímicos de alta resolución con cantidades muy reducidas de muestra. Una de las aplicaciones más prometedoras de la tecnología de órganos-en-chips es servir como un tipo de análisis pre-clínico complementario a los estudios en animales, para generar las predicciones necesarias antes de realizar los estudios clínicos en humanos. En la actualidad, uno de los retos que enfrenta la industria farmacéutica es poder disminuir los costos y hacer más eficiente la investigación y el desarrollo de nuevos fármacos con aplicación clínica, por lo cual, estos sistemas biomiméticos pueden ser usados como modelos especializados in vitro para facilitar la investigación mecanística y la modulación farmacológica de muchos procesos biológicos. En el futuro, estos dispositivos podrán ser usados en la medicina personalizada y en estudios clínicos, en donde las propias células del paciente podrán ser cultivadas en estos dispositivos en una especie de humano-en-un-chip (human-on-a-chip), para así estudiar la seguridad en el uso de los fármacos, su eficacia y los efectos colaterales que pudieran tener en cada individuo. Cabe mencionar que el avance en este tipo de desarrollos tecnológicos dependerá no sólo del esfuerzo de los científicos, sino también de los acuerdos que se establezcan entre las instituciones académicas, la industria y el gobierno. Así, se espera que muy pronto los dispositivos del tipo órganos-en-un-chip, cuerpo-en-un-chip, e incluso organismo-en-un-chip sean de las tecnologías con mayores aplicaciones en la biomedicina.

\section{REFERENCIAS}

1. Bhatia SN, Ingber DE. Microfluidic organs-on-chips. Nat Biotechnol. 2014; 32 (8): 760-772.

2. Derkus B. Applying the miniaturization technologies for biosensor design. Biosensor Bioelectron. 2016; 79: 901-913.

3. Esch EW et al. Organs-on-chips at the frontiers of drug discovery. Nat Rev Drug Discov. 2015; 14 (4): 248-260.

4. Hamon M, Hong JW. New tools and new biology: recent miniaturized systems for molecular and cellular biology. Mol Cells. 2013; 36 (6): 485-506.

5. Hautefeuille M. Jefe del Laboratorio Nacional de Sistemas Biomiméticos para Diagnóstico y Terapia, de la Facultad de Ciencias de la UNAM. LANSBioDyT: Available in: https://sites.google.com/a/ciencias.unam.mx/lansbiodyt/home

6. Materne EM et al. The multi-organ chip - a microfluidic platform for long-term multi-tissue coculture. J Vis Exp. 2015; 98: e52526. 
7. Paguirigan AL, Beebe DJ. From the cellular perspective: exploring differences in the cellular baseline in microscale and microfluidic cultures. Integr Biol (Camb). 2009; 2: 182-195.

8. Perestrelo AR et al. Microfluidic organ/body-on-a-chip devices at the convergence of biology and microengineering. Sensors (Basel). 2015; 12: 31142-31170.

9. Polacheck WJ et al. Microfluidic platforms for mechanobiology. Lab Chip. 2013; 13: 2252-2267.
10. Vázquez-Victorio G et al. Chapter 9: GPCRs and actin-cytoskeleton dynamics. Methods in Cell Biology. 2016; 132. Elsevier, Inc. (En prensa).

Dirección para correspondencia:

Marina Macías Silva

E-mail:mmacias@ifc.unam.mx 